\title{
PHASE-FIELD SIMULATION DURING RECRYSTALLIZATION PROCESS COUPLED WITH CRYSTAL PLASTICITY THEORY
}

\author{
Tomohiro Takaki", Akinori Yamanaka ${ }^{* *}$, Yoshikazu Higa ${ }^{* * *}$, Yoshihiro Tomita ${ }^{* * * *}$ \\ *Faculty of Maritime Sciences, Kobe University \\ Fukaeminami, Higashinada, Kobe 658-0022, Japan, \\ takaki@maritime.kobe-u.ac.jp \\ **,**** Graduate School of Science and Technology, Kobe University \\ Rokkodai, Nada, Kobe 657-8501, Japan, \\ ***yamanaka@solid.mech.kobe-u.ac.jp, ${ }^{* * * * *}$ tomita@mech.kobe-u.ac.jp \\ **** Department of Mechanical System Engineering, Okinawa National College of \\ Technology, \\ Henoko, Nago 905-2192, Japan, \\ y.higa@okinawa-ct.ac.jp
}

\begin{abstract}
A phase-field model and computational procedure for primary static recrystallization are developed to study the recrystallization kinetics. The deformation microstructures and plastic deformation behaviors of polycrystalline metal are simulated by finite element method using strain gradient crystal plasticity theory. Based on the results of crystallographic orientation and dislocation density, recrystallization phasefield simulation is performed. The effects of nucleation model of recrystallized grain on the recrystallization kinetics are also evaluated.
\end{abstract}

INTRODUCTION: It is well known that the key to controlling properties of metallic material is to control microstructure. It is, therefore, very important to understand the relationships between processing parameters and microstructural evolution. Monte Carlo Potts models [Srolovitz et al. 1986] and cellular automata models [Hesselbarth et al. 1991, Raabe 2002] are widely used as numerical models for static recrystallization. The both models are very similar each other and describe the state in terms of discrete values. The MC Potts model requires an introduction of spatial and kinetic scaling, because time and space in MC simulation is not real. On the other hand, although cellular automata model is more efficient and easier to extend to 3D problem than MC model, it does not allow for curvature as a driving force for migration.

Phase-field models have attracted considerable interest in the last decade as a means of simulating complicated microstructures. It does not require any scaling because of treating real time and space, and the curvature effect is included in the model explicitly. We, therefore, believe that the phase-field model is promising approach to simulate recrystallization. In this study, we develop a phase-field model and computational procedure of primary static recrystallization. We adopt an adaptive mesh technique to perform the phase-field simulation efficiently [Takaki et al. 2005]. The crystallographic orientation and dislocation density of plastically deformed material, which are required as driving force of recrystallized grain and information of nucleation site, are calculated by 
strain gradient crystal plasticity finite element method [Higa et al. 2003]. Some recrystallization simulations are conducted to compare nucleation models, because the nucleation condition is considered to be dominant factor that decide the recrystallization microstructures.

PROCEDURES, RESTULTS AND DISCUSSION: The procedure for primary static recrystallization developed here consists of three steps. First, the deformation behavior is evaluated by finite element method using strain gradient crystal plasticity theory. Second, the results, such as the crystallographic orientation, accumulated dislocation density and stored energy, are transferred into phase-field method. Finally, phase-field simulation is performed during isothermal annealing to simulate the nucleation and evolution of recrystallized grains.

Figure 1 shows the results of crystal plasticity finite element simulation, where polycrystalline aluminum with regular hexagonal grains is compressed $45 \%$. The black lines are original grain boundaries. The distribution of dislocation density $\rho\left(\rho_{\min }=0.30\right.$, $\left.\rho_{\max }=1.47\left[\times 10^{15} 1 / \mathrm{m}^{2}\right]\right)$ and high angle grain boundaries in which misorientation is larger than $15^{\circ}$ are illustrated in Fig.1. These data are transferred into phase-field simulation and used for calculating a stored energy and used as information of nucleation.

In recrystallization phase-field simulation, we solve following TDGL equations for phase field $\phi$ and crystallographic orientation $\theta$ :

$$
\frac{\partial \phi}{\partial t}=M_{\phi}\left[\alpha^{2} \nabla^{2} \phi-\frac{\partial f(\phi)}{\partial \phi}-2 \phi s|\nabla \theta|\right] \quad(1), \quad \frac{\partial \theta}{\partial t}=M_{\theta} \frac{1}{\phi^{2}} \nabla \cdot\left(\phi^{2} s \frac{\nabla \theta}{|\nabla \theta|}\right)
$$

where phase field $\phi$ equals zero in the deformed matrix and unity in recrystallized grain, and have $0<\phi<1$ inside recrystallized grain boundary. $f(\phi)$ is a free energy density and expressed by double well type function: $f(\phi)=(1-p(\phi)) E_{\text {store }}+W q(\phi)$ where $p(\phi)=\phi^{3}\left(10-15 \phi+6 \phi^{2}\right), \quad q(\phi)=\phi^{2}(1-\phi)^{2}, \quad E_{\text {store }}=0.5 \rho \mu \tilde{b}^{2}$ stored energy, and $W=6 \sigma / \delta$ energy wall height related to interface energy $\sigma$ and interface thickness $\delta$. Furthermore, $\alpha=\sqrt{3 \delta \sigma}$ gradient coefficient and $s=\alpha \sqrt{2 W} / \pi \cdot M_{\phi}=m \sqrt{2 W} / 6 \alpha$ and $M_{\theta}=(1-p(\phi)) M_{\phi}+p(\phi) M_{\phi} / 1000$ are mobilities for $\phi$ and $\theta$, respectively, where $m$ is a mobility of grain boundary migration. Here we consider three types of nucleation model, i.e. site saturated nucleation (nuclei are placed at $t=0$ on area satisfying $\Delta \theta>15^{\circ}$ and $\rho>$ $a \rho_{\max }$ where $a=0.5,0.6$ and 0.7 ), constant nucleation rate (one nucleus is placed every $10,100,500$ and 1000 time steps) and decreasing nucleation rate (constant nucleation rate for unrecrystallized area). Figure 2 shows time evolutions of recrystallized grains and adaptive mesh, and final recrystallized microstructures for decreasing nucleation rate model (100 steps). Comparing with Fig.1, we can see from Fig.2 that the nuclei occur at high dislocation density area and on the high angle boundaries. Figure 3 indicates the recrystallized area fraction $X$ versus time for three nucleation models. 

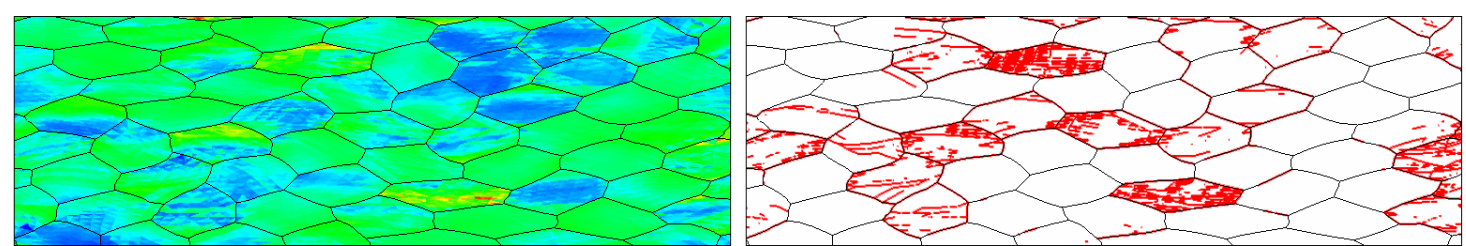

Fig. 1 Distributions of dislocation density and high angle grain boundary at $45 \%$ compression of polycrystalline aluminum

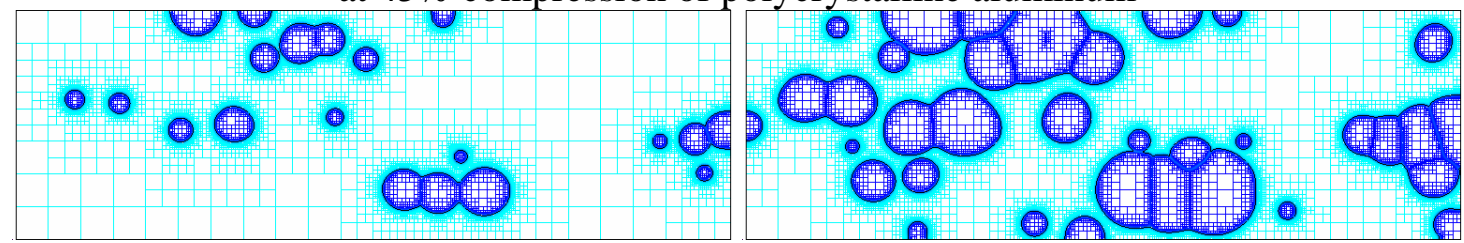

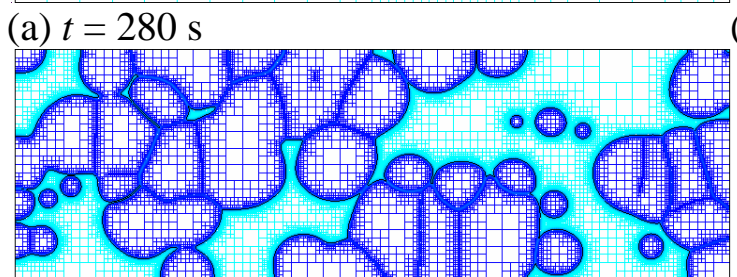

(c) $t=840 \mathrm{~s}$ (b) $t=560 \mathrm{~s}$

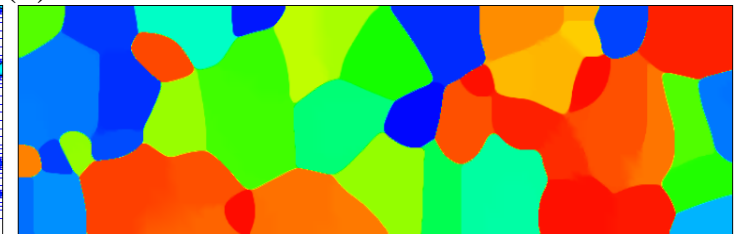

(d) $t=1820 \mathrm{~s}$

Fig. 2 Time evolutions of recrystallized grain (blue region) and adaptive mesh, and final recrystallized microstructure (color indicates crystallographic orientation).
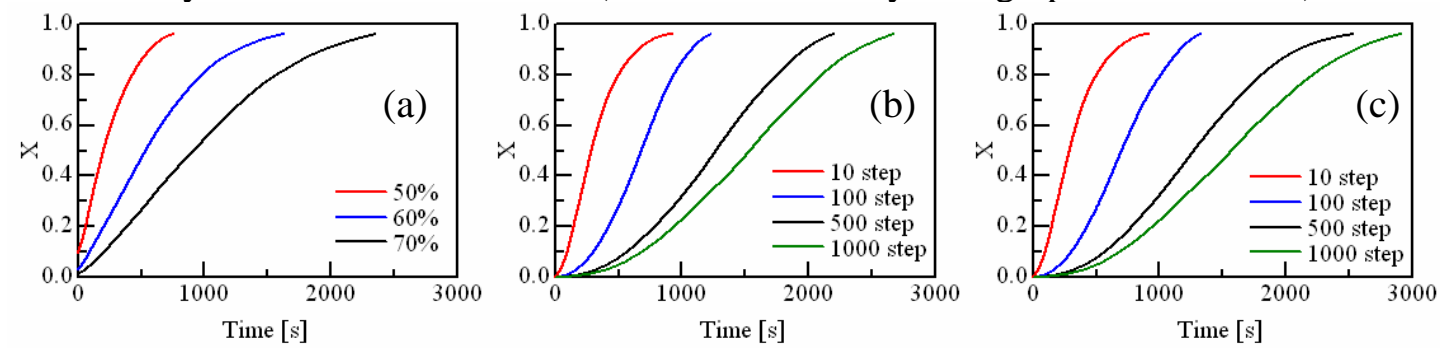

Fig.3 Recrystallized area fraction vs time for (a) site saturated nucleation, (b) constant nucleation rate and (c) decreasing nucleation rate.

\section{REFERENCES:}

Srolovitz D. J., Grest G. S. and Anderson M. P., 1986, "Computer Simulation of Recrystallization - I. Homogeneous Nucleation and Growth", Acta metal. 34, 18331845 .

Hasselbarth H. W. and Göbel I. R., 1991, "Simulation of Recrystallization by Cellular Automata", Acta metal. mater. 39, 2135-2143.

Raabe D., 2002, "Cellular Automata in Materials Science with Particular Reference to Recrystallization Simulation”, Annu. Rev. Mater. Res. 32, 53-76.

Takaki T., Fukuoka T. and Tomita Y., 2005, "Phase-field simulation during directional solidification of a binary alloy using adaptive finite element method", J. Crystal Growth 283, 263-278.

Higa Y., Sawada Y. and Tomita Y., 2003, "Computational Simulation of Characteristic Length Dependent Deformation Behavior of Polycrystalline Metals", Tran. JSME 69, 523-529. 